\title{
An Autopsy Case of a Nonprescription Aspirin Overdose and Chlorine Gas Exposure
}

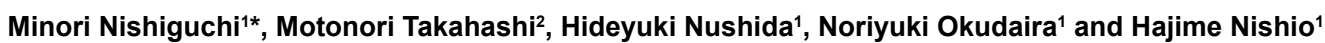

${ }^{1}$ Department of Legal Medicine, Hyogo College of Medicine, Nishinomiya, Japan

${ }^{2}$ Division of Legal Medicine, Department of Community Medicine and Social Healthcare Science, Kobe University Graduate School of Medicine/School of Medicine, Kobe, Japan

\begin{abstract}
We report an autopsy case of a man whose death was caused by a combination of aspirin (acetylsalicylic acid) poisoning and chlorine gas inhalation. Toxicological analysis of aspirin and salicylic acid using high performance liquid chromatography with photodiode array detection showed that the concentration of salicylic acid was $444.7 \mu \mathrm{g} /$ $\mathrm{mL}$ in the heart blood and $68.3 \mu \mathrm{g} / \mathrm{g}$ in the brain. Existence of chlorine gas was proven, in the form of chloride ions, in a plastic bag near the body using energy dispersive X-ray fluorescence spectrometry. It was thought that damage to the mucosa, respiratory tract and skin had been caused by hydrochloric and hypochlorous acid associated with chlorine gas inhalation that rapidly aggravated the respiratory distress and pulmonary edema caused by aspirin and salicylic acid. This case report shows that the risk of death is increased when two substances, aspirin and chlorine gas, are taken through different routes, even if the concentration of one of them does not reach the lethal dose.
\end{abstract}

Keywords: Aspirin; Salicylic acid; Chlorine gas; Overdose; Toxicity

\section{Introduction}

Aspirin (acetylsalicylic acid), with fewer adverse reactions than salicylic acid, was first generated as an antipyretic analgesic through acetylation of salicylic acid in the late $19^{\text {th }}$ century [1-3]. Since then, aspirin has long been used as an ingredient in anti-inflammatory agents worldwide and is commonly used as a drug with antiinflammatory, antipyretic and analgesic effects, as well as antiplatelet and antirheumatic effects [4-6]. Aspirin has been used as a prescription drug and nonprescription (over-the-counter) drug, and demand for the drug has steadily increased [6,7]. However, some nonprescription drugs that are formulated with a large amount of aspirin and no antacid have potent effects, which raise concern about the use of these products. Consequently, cases of aspirin poisoning caused by overdose are common [8-10].

We report an autopsy case of fatal poisoning by chlorine gas inhalation and ingestion of a large amount of nonprescription aspirin. Toxicological analysis showed high concentrations of the active metabolite of aspirin, salicylic acid, in the blood and brain. Metabolic acidosis that is characteristic of salicylism increases the distribution of salicylic acid into the brain.

Furthermore, high doses of aspirin prolong the half-life of salicylic acid, resulting in toxicity, such as pulmonary edema. In addition, damage to the lungs and respiratory tract may be aggravated by chlorine inhalation. The possibility that the male died from aspirin and chlorine gas poisoning is high.

\section{Case Report}

\section{Case history}

A 48-year-old male (height $168 \mathrm{~cm}$, weight $53.7 \mathrm{~kg}$ ) was found dead in a hotel bed. Two empty toilet detergent bottles and one kitchen bleach bottle were found at the bedside on the floor. A plastic bag containing a clear and colorless liquid, which was assumed a mixture of toilet detergent and kitchen bleach, was found beside the decedent. Moreover, six empty cartons (total 60 tablets) of nonprescription analgesic antipyretics $\left(\right.$ Aspirin $\left.^{\circledR}\right)$ were in the garbage can. He had no past medical history. To clarify the cause of death, a forensic autopsy was performed approximately $36 \mathrm{~h}$ after death.

\section{Autopsy findings}

The decedent was $168 \mathrm{~cm}$ tall and weighed $53.7 \mathrm{~kg}$. The face showed red-brown discoloration with erosion, especially on the skin around the lips with foam exuding from the mouth following pulmonary edema displayed severe erosion. The external findings were unremarkable. Internally, the left and right lungs weighed 1100 and $1000 \mathrm{~g}$, respectively, and showed marked pulmonary edema and congestion. The trachea and bronchi were filled with froth, and many ecchymoses were observed in the mucosa. There was approximately $150 \mathrm{~mL}$ of stomach contents containing a reddish-brown liquid that included a white granule. The heart weighed $270 \mathrm{~g}$ and contained approximately $200 \mathrm{~mL}$ of dark red and viscous blood. Femoral blood could not be collected for a blood clot. The coronary artery showed no abnormal findings including stenosis. The brain weighed $1300 \mathrm{~g}$, and surface was slightly filling. Most organs showed slight congestion. Postmortem samples including heart blood, urine, stomach contents, organs (brain, lung and liver) and the liquid in the plastic bag were collected during autopsy and kept at $-40^{\circ} \mathrm{C}$ for toxicological examination.

\section{Toxicological Examination}

\section{Materials}

Aspirin, salicylic acid and 2-methylbenzoic acid (internal standard;

*Corresponding author: Minori Nishiguchi, Department of Legal Medicine, Hyogo College of Medicine 1-1 Mukogawa-cho, Nishinomiya, Hyogo 663-8501, Japan, Tel: +81-798-45-6578; Fax : +81-798-49-3279; E-mail: m-nishig@hyo-med.ac.jp

Received July 06, 2013; Accepted August 13, 2013; Published August 21, 2013

Citation: Nishiguchi M, Takahashi M, Nushida H, Okudaira N, Nishio H (2013) An Autopsy Case of a Nonprescription Aspirin Overdose and Chlorine Gas Exposure. J Forensic Res 4: 187. doi:10.4172/2157-7145.1000187

Copyright: (c) 2013 Nishiguchi M, et al. This is an open-access article distributed under the terms of the Creative Commons Attribution License, which permits unrestricted use, distribution, and reproduction in any medium, provided the original author and source are credited. 
IS) were purchased from Wako Pure Chemicals (Osaka, Japan). All the other reagents and solvents for instrumental analysis were analytical grade. The formalin solution used for tissue fixat ion was purchased from Wako Pure Chemicals (Osaka, Japan).

\section{Sample preparation}

Analysis of aspirin and salicylic acid in various fluid and organ samples was performed in accordance with the modified Needs' method [11]. In brief, $200 \mu \mathrm{L}$ fluid samples were added to $200 \mu \mathrm{L}$ internal standard (5 $\mu \mathrm{l} / \mathrm{mL}$ 2-methylbenzoic acid). After agitation, $400 \mu \mathrm{L}$ acetonitrile was added drop wise slowly to the sample solution. After the mixture was centrifuged $(20,630 \mathrm{xg}, 10 \mathrm{~min}$; KUBOTA 3300 , Tokyo, Japan), $20 \mu \mathrm{L}$ of the supernatant was injected into an HPLC-PDA. The organ samples $(0.5 \mathrm{~g})$ were homogenized with $1 \mathrm{~mL}$ distilled water and centrifuged for $10 \mathrm{~min}$ at 2,270 $\mathrm{xg}$ (KUBOTA 5910). Following centrifugation, $200 \mu \mathrm{L}$ of the supernatant was deproteinized with acetonitrile $(400 \mu \mathrm{L})$. After the mixture was centrifuged $(20,630$ $\mathrm{xg}, 10 \mathrm{~min}$; KUBOTA 3300), $20 \mu \mathrm{L}$ of the supernatant was injected into the HPLC-PDA.

For energy dispersive X-ray fluorescence spectrometric analysis, the fluid and organ samples of this case and 10 samples of non-drug cases (control samples) were analyzed without any further sample preparation.

\section{Instrumentation}

Quantification of aspirin (i.e., acetylsalicylic acid) and salicylic acid were performed using high performance liquid chromatography with photodiode array detection (HPLC-PDA; Class-VP system, Shimadzu, Kyoto, Japan). The HPLC-PDA system consisted of a pump (LC-10ADvp) and detector (both from Shimadzu). Analysis of drugs was performed using a Develosil ODA-UG-5 column (4.6 $\mathrm{mm}$ i.d. $\times 250 \mathrm{~mm})$ and Shim-pack SPC-RP3 column $(4.0 \mathrm{~mm}$ i.d. $\times 30 \mathrm{~mm})$ at $50^{\circ} \mathrm{C}$. The mobile phases used were $2.8 \mathrm{mM}$ phosphate buffer solution ( $\mathrm{pH}$ 2.7) and acetonitrile. The gradient program of the mobile phase was performed as follows: $10 \%$ acetonitrile at $0 \mathrm{~min}$, $50 \%$ at $10 \mathrm{~min}$ with a constant flow rate of $1.0 \mathrm{~mL} / \mathrm{min}$. The chlorine gas (in the form of chloride ion) of the postmortem samples and the clear and colorless liquid in the plastic bag were analyzed by energy dispersive X-ray fluorescence spectrometry (EDXRF; JSX-3220, JEOL, Tokyo, Japan). EDXRF is a method that detects fluorescence $\mathrm{X}$-rays emitted from a sample following X-irradiation. The type and concentration of an element that are contained in a sample can be determined nondestructively. This case indicates that screening with EDXRF provides useful information for drug identification [12]. The operating conditions for EDXRF were as follows: target: $\mathrm{Rh}$ anode; operating voltage and current: $30 \mathrm{kV} / 300 \mu \mathrm{A}$; detector: silicon-lithium; and measurement time: $300 \mathrm{sec}$. The blood and urine samples were analyzed for ethanol by headspace gas chromatography (Auto System XL, PerkinElmer and Waltham, MA, USA).

\section{Method validation}

To plot calibration curves by HPLC-PDA, body fluid and organ samples spiked with the standard drugs at five different concentrations (from $0.1-1,000 \mu \mathrm{g} / \mathrm{mL}$ ) were analyzed according to the previously described procedure. The calibration curve was obtained by plotting the peak area ratios of each standard drug to IS against drug concentration. The limit of detection and the limit of quantification were 0.1 and 1.0 $\mu \mathrm{g} / \mathrm{mL}$, respectively, for aspirin and salicylic acid in body fluid and organ samples $(\mathrm{S} / \mathrm{N}=3)$.

\section{Histological examination}

The brain and lung tissue samples were fixed in $10 \%$ formalin solution and embedded in paraffin. Serial paraffin sections, $3-\mu \mathrm{m}$ thick, were prepared and stained with hematoxylin and eosin. The stained sections were evaluated under different magnifications of light microscopy (Nikon Eclipse 50i, Nikon Corp., and Tokyo, Japan).

\section{Results}

The results of the toxicological analysis of this case are presented in Table 1. In all body fluid and organ samples, salicylic acid was detected in relatively high concentrations, and chloride ion levels were nearly equivalent to the control group. In the clear and colorless liquid contained in the plastic bag, aspirin and salicylic acid were not identified; chloride ion was detected in large quantities. No ethanol was detected in the blood or urine. Drug screening results using the Triage ${ }^{\mathbb{R}}$ Drugs of Abuse Panel (Biosite Diagnostic Inc., San Diego, CA,USA) were negative. Histopathological examination revealed pulmonary edema (Figure 1). However, cerebral edema was not observed.

\section{Discussion}

In this case, aspirin and its active metabolite, salicylic acid, were detected in heart blood at concentrations of 1.0 and $444.7 \mu \mathrm{g} / \mathrm{mL}$, respectively. Aspirin has an elimination half-life of 15 to 20 minutes and is rapidly hydrolyzed to salicylic acid by blood and liver esterases [11-15]. Salicylic acid at a dose of $3.0 \mathrm{~g}$ or less is relatively rapidly eliminated from the blood with an elimination half-life of 2 to 4 hours. However, when large amounts of aspirin are ingested $(>10 \mathrm{~g})$, the elimination half-life is prolonged to 20 hours or more [11,15,16], and absorption may be delayed due to salicylate-induced pylorospasm and/ or the formation of pharmacobezoars [16]. In this case, we estimate that the deceased took approximately 60 tablets of non-prescription aspirin products (500 mg/tablet), and therefore, salicylic acid remained in the blood at a high concentration for a relatively long time. Poisoning caused by intake of large amounts of aspirin is known as salicylism. In this situation, metabolic acidosis and respiratory alkalosis develop

\begin{tabular}{|c|c|c|c|}
\hline Specimen & Acetylsalicylic acid & Sal icylic acid & Chloride ion (vs control group) \\
\hline Heart blood & 1.0 & 444.7 & 105.9 \\
\hline Urine & ND & 5118.1 & Equal \\
\hline Stomach contents & 3115.5 & 68.3 & Equal \\
\hline Brain & ND & 60.9 & Equal \\
\hline Lung & ND & 34.5 & Equal \\
\hline Liver & ND & ND & Equal \\
\hline Colorless transparent liquid & ND & Very large quantity \\
\hline
\end{tabular}

ND: not detectable at the limits of quantitation of $1.0 \mu \mathrm{g} / \mathrm{mL}$ o $\mathrm{r} \mu \mathrm{g} / \mathrm{g}$ for body fluid and organ samples

Table 1: Acetylsal icylic acid and salicyl ic acid concentration in fluid and organ samples $(\mu \mathrm{g} / \mathrm{mL}$ or $\mu \mathrm{g} / \mathrm{g})$. Chloride ion was indicated the relat ive amount compared to the control group. 


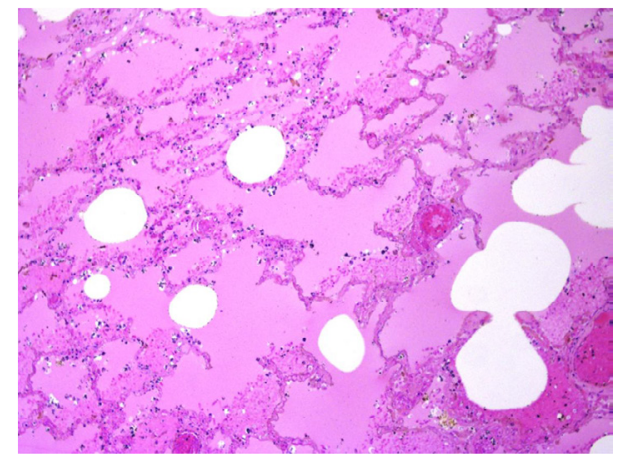

Figure 1: Histopathology of pulmonary evidence severed edema (H\&E stain magnification $\mathrm{X} 10$ )

due to a serious acid-base imbalance and lead to heart and respiratory failure, resulting in death [17]. The pathological mechanism is direct stimulation of the respiratory center in the medulla oblongata by salicylate, with hyperpnea and hyperventilation decreasing the blood $\mathrm{CO}_{2}$ concentration and causing respiratory alkalosis [18]. Subsequently, salicylate distributed into cells from the blood directly inhibits oxidative phosphorylation in these cells, which reduces ATP production and causes an imbalance of metabolism and energy supply, resulting in metabolic acidosis [19]. In this case, salicylic acid was present at levels of $68.3,60.9$ and $34.5 \mu \mathrm{g} / \mathrm{g}$ in the brain, lungs and liver, respectively. These values are lower than those in previous cases of death from aspirin poisoning $(300-400,900-1400$, and $600-800 \mu \mathrm{g} / \mathrm{g}$ in the brain, lungs and liver, respectively) [20]. However, our case notes that the level in the brain was greater than those in the lungs and liver. Salicylic acid is an acidic substance that is charged in the blood and is unlikely to pass through the lipid bilayer of the blood-brain barrier [20,21]. However, when large amounts of aspirin are taken, metabolic acidosis is induced, and the high concentration of salicylic acid decreases blood $\mathrm{pH}$. This increases the undissociated form of salicylic acid and allows the drug to pass through the blood-brain barrier. Distribution of salicylic acid to the brain causes cerebral edema [22] and has a central nervous system effect on the respiratory center [18,23] regardless of the blood concentration. Therefore, although it is not clear which of respiratory alkalosis or metabolic acidosis affected to his death, it is certainly that the high concentration of salicylic acid in our case also had a serious effect on the central nervous system.

The lethal dose of aspirin is $>500 \mathrm{mg} / \mathrm{kg}$ body weight [19]. The possibility of death exists in this case, where the decedent weighed 53.7 $\mathrm{kg}$ and took at least 54 aspirin tablets $(500 \mathrm{mg} /$ tablet). Further, the effects of chlorine gas may have been ancillary to the lethal effect of aspirin. It is thought that chlorine gas was generated by mixing toilet detergent (hydrochloric acid) with kitchen bleach (sodium hypochlorite) in the plastic bag. Chlorine gas has direct effects on humans, which mainly include strong irritation of the mucosa of the eyes, nose and upper and lower respiratory tract and dermal inflammation [24,25]. In this case, the erosion of the face and around the lips, and severe pulmonary edema suggested that damage to the skin and mucosa of the respiratory tract was caused by chlorine gas inhalation. Furthermore, inhaled chlorine gas dissolves in the mucus in the respiratory tract and alveoli, and subsequently generates hydrochloric and hypochlorous acid that destroy the cellular structure. Erosion around the lips was most likely due to a chemical burn caused by contact with froth containing these acids. This eliminated the possibility of intake of the clear and colorless liquid because chloride ions were not detected in the heart blood, urine and gastric contents. However, it was thought that damage to the mucosa, respiratory tract and skin had been caused by hydrochloric and hypochlorous acid associated with chlorine gas inhalation that rapidly aggravated the respiratory distress and pulmonary edema caused by aspirin and salicylic acid.

In summary, we examined a deceased person who inhaled chlorine gas and ingested a large number of aspirin tablets. The active metabolite of aspirin, salicylic acid, was detected at a high concentration in the blood and in several organs, and especially in the brain. In addition, mucosal irritation of the respiratory tract was produced by inhalation of a high concentration of chlorine gas. Consequently, we conclude that the cause of death was salicylic acid poisoning combined with chlorine gas inhalation. This case demonstrates that the risk of death is increased when two substances are taken through different routes, even if the concentration of one of them does not reach the lethal dose. This is particularly true if one of the substances is a toxic gas that causes pulmonary damage.

\section{References}

1. Vane JR, Botting RM (2003) The mechanism of action of aspirin. Thromb Res 110: $255-258$

2. Jóhannesson T (2000) [Aspirin. Acetylsalicylic acid and aspirinlike drugs. A review.] Laeknabladid 86: 755-768.

3. Vainio H, Morgan G (1997) Aspirin for the second hundred years: new uses for an old drug. Pharmacol Toxicol 81: 151-152.

4. Lee P, Anderson JA, Miller J, Webb J, Buchanan WW (1976) Evaluation of analgesic action and efficacy of antirheumatic drugs. Study of 10 drugs in 684 patients with rheumatoid arthritis. J Rheumatol 3: 283-294.

5. Pongrácz E, Káposzta Z (2005) Antiplatelet therapy in ischemic stroke. Expert Rev Neurother 5: 541-549.

6. Rainsford KD (2007) Anti-inflammatory drugs in the 21st century. Subcell Biochem 42: 3-27.

7. Clissold SP (1986) Aspirin and related derivatives of salicylic acid. Drugs 32 Suppl 4: 8-26.

8. Elkind AH (1991) Drug abuse and headache. Med Clin North Am 75: 717-732.

9. Jones A (2002) Over-the-counter analgesics: a toxicology perspective. Am J Ther 9: 245-257.

10. Herres J, Ryan D, Salzman M (2009) Delayed salicylate toxicity with undetectable initial levels after large-dose aspirin ingestion. Am J Emerg Med 27: 1173.

11. Needs CJ, Brooks PM (1985) Clinical pharmacokinetics of the salicylates. Clin Pharmacokinet 10: 164-177.

12. Takahashi M, Kinoshita $H$, Nishiguchi M, Kasuda S, Ouchi $H$, et al. (2009) Application of energy dispersive X-ray fluorescent spectrometry (EDXRF) in drug-related cases. Leg Med (Tokyo) 11 Suppl 1: S411-412.

13. Thiessen JJ (1983) Aspirin: plasma concentration and effects. Thromb Res Suppl 4: 105-111.

14. Trnavsky K, Zachar M (1975) Correlation of serum aspirin esterase activity and half-life of salicylic acid. Agents Actions 5: 549-552.

15. Kamanyire R (2002) Aspirin overdose. Emerg Nurse 10: 17-22.

16. Rivera W, Kleinschmidt KC, Velez LI, Shepherd G, Keyes DC (2004) Delayed salicylate toxicity at 35 hours without early manifestations following a single salicylate ingestion. Ann Pharmacother 38: 1186-1188.

17. Tschetter PN (1963) SALICYLISM. Am J Dis Child 106: 334-346.

18. Brem J, Pereli EM, Gopalan SK, Miller TB (1973) Salicylism, hyperventilation, and the central nervous system. J Pediatr 83: 264-266.

19. Temple AR (1981) Acute and chronic effects of aspirin toxicity and their treatment. Arch Intern Med 141: 364-369. 
Citation: Nishiguchi M, Takahashi M, Nushida H, Okudaira N, Nishio H (2013) An Autopsy Case of a Nonprescription Aspirin Overdose and Chlorine Gas Exposure. J Forensic Res 4: 187. doi:10.4172/2157-7145.1000187

Page 4 of 4

20. Ihama Y, Ageda S, Fuke C, Miyazaki T (2007) [Autopsy case of aspirin intoxication: distribution of salicylic acid and salicyluric acid in body fluid and organs]. Chudoku Kenkyu 20: 375-380.

21. Levin VA (1980) Relationship of octanol/water partition coefficient and molecular weight to rat brain capillary permeability. J Med Chem 23: 682-684.

22. Bray PF, Gardiner AY (1977) Salicylism and severe brain edema. N Engl J Med 297: 1235.
23. Buchanan N, Kundig H, Eyberg C (1975) Experimental salicylate intoxication in young baboons. A preliminary report. J Pediatr 86: 225-232.

24. Cevik Y, Onay M, Akmaz I, Sezigen S (2009) Mass casualties from acute inhalation of chlorine gas. South Med J 102: 1209-1213.

25. Gorguner M, Aslan S, Inandi T, Cakir Z (2004) Reactive airways dysfunction syndrome in housewives due to a bleach-hydrochloric acid mixture. Inhal Toxicol 16: 87-91. 